\title{
Macroscopic Resonant Tunneling of Magnetization in Ferritin
}

\author{
J. Tejada, X. X. Zhang, E. del Barco, and J. M. Hernández \\ Departamento de Fisica Fonamental, Universitat de Barcelona, Diagonal 647, Barcelona, 08028, Spain \\ E. M. Chudnovsky \\ Physics Department, Lehman College, The City University of New York, Bronx, New York 10468-1589
}

(Received 16 April 1997)

\begin{abstract}
We report experimental evidence of macroscopic quantum tunneling in antiferromagnetic particles. The zero-field maximum in the dependence of the magnetic relaxation rate on the magnetic field in ferritin has been observed. This observation provides a new insight into the origin of the nonmonotonic field dependence of the blocking temperature reported recently by Gider et al. [J. Appl. Phys. 79, 5324 (1996)] and by Friedman et al. (unpublished). We argue that the effect comes from the resonant spin tunneling between matching spin levels and cannot be due to the interparticle interactions. Our findings are in agreement with the tunneling interpretation of earlier experiments in ferritin by Awschalom et al. [Phys. Rev. Lett. 68, 3092 (1992)]. [S0031-9007(97)03945-8]
\end{abstract}

PACS numbers: 75.45. $+\mathrm{j}, 75.50 . \mathrm{Tt}, 75.60 . \mathrm{Lr}$

Quantum dynamics of the magnetization in ferritin has received much attention since Awschalom and co-workers reported the existence of a low temperature resonance in the absorption spectrum of horse spleen ferritin, which they attributed to the macroscopic quantum coherence [1]. Ferritin is an iron storage protein. It has a spherical cage of about $8 \mathrm{~nm}$ in diameter that contains the mineral ferrihydrite combined with a phosphate. Its core is equivalent to a small antiferromagnetic particle. The size of the core in natural ferritin ranges from 3 to $7.5 \mathrm{~nm}$. The fully packed ferritin contains $4500 \mathrm{Fe}^{3+}$ ions. A small magnetic moment of the particle arises from the noncompensation of collinear spin sublattices due to the finite size and irregular shape of the core. The spin of the sublattice $S$ is of the order of 5000, while the noncompensated spin $s$ is below 100. This noncompensated spin looks in one of two directions along the anisotropy axis of the particle. Above $13 \mathrm{~K}$ the particles are superparamagnetic [2]; that is, their moments frequently jump between the two orientations over the anisotropy barrier. Well below $13 \mathrm{~K}$ thermal processes are essentially frozen and the transition from $\mathbf{s}$ to $-\mathbf{s}$ can occur only due to quantum tunneling: in the absence of the magnetic field and in the limit of weak dissipation, sublattices should interchange in a coherent manner. It has been demonstrated [3] that quantum tunneling of the magnetization in antiferromagnets is much stronger than in ferromagnets [4-6]. Achieving a narrow distribution of the magnetic core sizes could then enable one to observe a resonance in the ac susceptibility and noise spectrum at the frequency of the ac field that coincides with the frequency of the coherent oscillations of the antiferromagnetic sublattices [1,7]. The additional, though indirect, evidence of spin tunneling in natural ferritin came from the observation of the temperature independent magnetic relaxation below $2.4 \mathrm{~K}$ [2]. There existed, however, a significant inconsistency, pointed out in Ref. [8], between high and low temperature behavior of narrowly distributed particles studied in Ref. [7]. While high temperature measurements indicated that magnetic moments of the particles were essentially frozen below a few kelvin, resonance experiments in the milikelvin range were interpreted in terms of spin tunneling at the rate $10^{6}-10^{9} \mathrm{~s}^{-1}$. A possible resolution of that paradox could be that the magnetization measurements in the kelvin range ignored the condition that spin levels in two potential wells separated by the anisotropy barrier should match to provide the high tunneling rate. The high rate observed in the $\mathrm{mK}$ range corresponded to the resonant spin tunneling, while the slow relaxation in the kelvin range was due to off resonance tunneling. If this is true, one should be able to observe the increase in the magnetic relaxation rate as the field approaches zero, which would be in apparent disagreement with classical behavior observed to date in many systems. In this paper we show that this is exactly what occurs in ferritin.

In our experiments we have used a diluted natural ferritin sample containing approximately $10^{14}$ ferritin molecules. At high enough temperature the molecules are superparamagnetic; their magnetic susceptibility follows the Curie law. The average magnetic moment of the molecule deduced from that law corresponds to 15 noncompensated $\mathrm{Fe}^{3+}$ ions. This is roughly in accordance with the number of noncompensated ions. Indeed, the number of ions at the surface is $\sim(4500)^{2 / 3}$. The square root of this number, $(4500)^{1 / 3} \sim 16$, gives an estimate of the number of noncompensated ions due to the surface randomness. Such a low moment means that the magnetic dipole interaction between the molecules is very weak.

The low temperature magnetization measurements followed the procedure described below. The sample was cooled down to $2 \mathrm{~K}$ from a high temperature superparamagnetic regime. Then a fixed field, ranging from 0.5 to $6 \mathrm{kOe}$, was applied and the dependence of the magnetization on temperature, the so-called zero-field cooled (ZFC) magnetization curves, was obtained (Fig. 1). The 


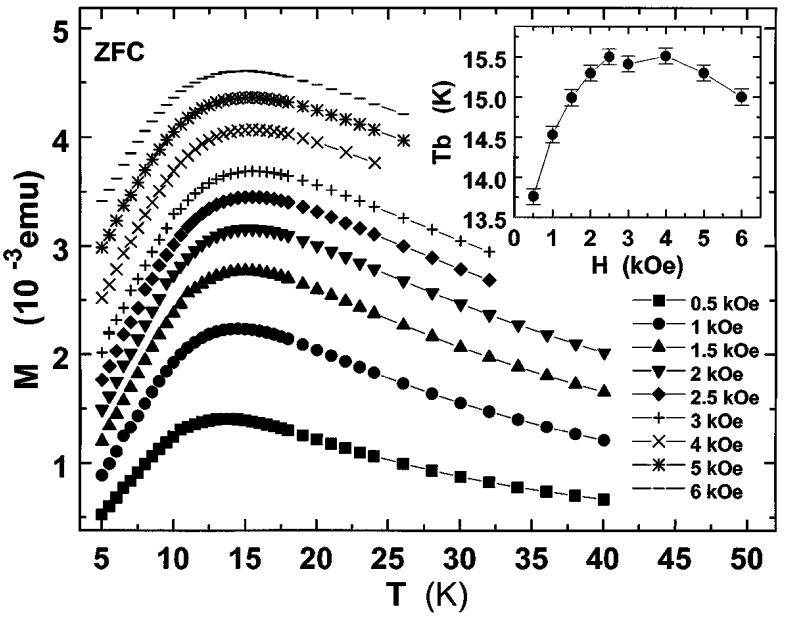

FIG. 1. Zero-field cooled magnetization curves obtained by cooling the sample in zero field and then heating it at different applied fields. The inset shows the field dependence of the blocking temperature.

maximum of the ZFC $M(T)$ curve determines the blocking temperature, $T_{B}$. The usual explanation of this curve in a system of small particles is this. At low temperature the rate at which individual moments of the particles jump across the anisotropy barrier $U$ is proportional to the Arrhenius factor, $\exp (-U / T)$. Natural ferritin particles are widely distributed over $U$. Because of the exponential dependence of the rate on $U$, at any temperature there is a rather sharp boundary between particles in which the moment is essentially frozen and particles in which the moment jumps between different orientations on the time scale of the experiment. As the temperature goes up, bigger and bigger particles unfreeze their moments, and the total $M$ in the direction of the field increases. Above $T_{B}$ moments of most of the particles are unfrozen and $M(T)$ approaches the Curie law. $T_{B}$ is, therefore, proportional to the barrier height in particles which mostly contribute to $M(T)$ on the time scale of the experiment $t \sim 10^{2} \mathrm{~s}$. Writing $U=K V$, where $K$ is the energy density of the magnetic anisotropy and $V$ is the volume of the particle, and $\tau=\tau_{0} \exp \left(K V / T_{B}\right)$, where $\tau_{0} \sim 10^{-10} \mathrm{~s}$ is the attempt time, we find that at $T_{B}=$ $13 \mathrm{~K}$ particles of the core diameter $7.5 \mathrm{~nm}$ must have $K=2.5 \times 10^{5} \mathrm{erg} / \mathrm{cm}^{3}$, which is a reasonable value for this class of antiferromagnets.

From the classical point of view, the magnetic field applied to a magnetic particle lowers the barrier between the directions of the moment along and against the field. Consequently, one should expect $T_{B}$ to decrease as the field increases. This is, in fact, a common behavior in systems of small particles. However, in ferritin the dependence of $T_{B}$ on $H$ is different. As is shown in the inset in Fig. 1, the blocking temperature has a nonmonotonic dependence on the field. It first increases until about $2.5 \mathrm{kOe}$ and then decreases at higher fields. The value of $T_{B}$ for each ZFC curve has been obtained with the accuracy within $0.1 \mathrm{~K}$ by computing the derivative $d M / d T$. The maximum in the dependence of $T_{B}$ on $H$ was established unambigously. This behavior of $T_{B}(H)$ in ferritin has also been observed by Gider et al. [9], by Friedman et al. [10] (whose work prompted our studies), and by Sappey et al. [11]. Additional support to the superparamagnetic origin of the effect comes from the measurements of the ac suceptibility. In these measurements, as compared to the dc ZFC magnetization measurements, the role of time is played by the frequency of the ac field, $f$. Correspondingly, the blocking temperature is determined by

$$
T_{B}=\frac{U}{\ln \left(1 / f \tau_{0}\right)} .
$$

The higher the frequency is the larger $T_{B}$ is. This allows one to conduct an independent test of whether the moments of the particles are frozen at low temperature by their individual anisotropy barriers or by interactions. In the latter case the blocking temperature must be a frequency independent glass temperature. The ac measurements show that $T_{B}$ in natural ferritin does depend on frequency in accordance with Eq. (1), providing an independent proof to the single-particle nature of the effect [12]. This conclusion is also supported by Mössbauer measurements [12] which correspond to frequencies as high as $f \sim 4 \times 10^{8} \mathrm{~Hz}$. Finally, one should consider the possibility that the ZFC magnetization curve is due to the evolution of a spin-glass state inside individual particles. Two experimental facts are against that picture and in favor of a simple superparamagnetic blocking mechanism. The first is the already mentioned frequency dependence of $T_{B}$ [12] which is in accordance with Eq. (1). The second is the linear dependence of $T_{B}$ on $V$, observed by Gider et al. [7], which is also in accordance with Eq. (1) and the relation $U=K V$.

As has been discussed above, the low temperature parts of the ZFC $M(T)$ curves in Fig. 1 reflect relaxation to the thermodynamic equilibrium. They suggest, though indirectly, that the approach to the equilibrium is faster at zero field than it is at a nonzero field in the range $0-2.5 \mathrm{kOe}$. Another way to see this is to plot the field derivative of the magnetization measured at a constant sweep rate. This method has been used to detect the resonant spin tunneling in Mn-12 molecules [13]. As is shown in Fig. 2, $d M / d H$ in ferritin has a sharp peak at $H=0$, which is very similar to the $H=0$ peak of $d M / d H$ in Mn-12. Finally, we confirmed the enhanced relaxation at $H=0$ in ferritin by measuring directly the rate of the magnetic relaxation as a function of the field. The following procedure has been applied to measure relaxation. The ferritin sample was cooled in zero field from $50 \mathrm{~K}$ down to the measurement temperature. The magnetic field was then applied and the sample was allowed to relax to the equilibrium state. With high accuracy the relaxation is logarithmic in time during four decades, as it should be in a system with broad 


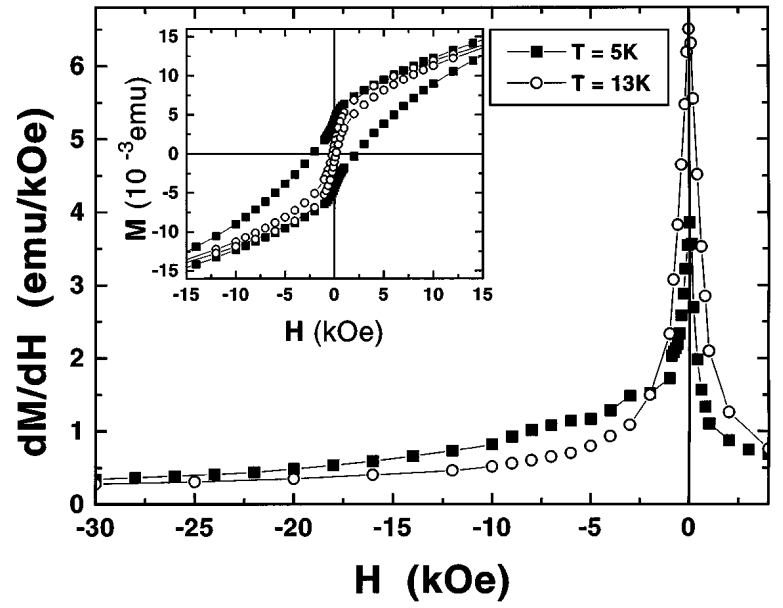

FIG. 2. Field derivative of the magnetization at a constant sweep rate. Inset shows hysteresis loops from which $d M / d H$ has been obtained.

distribution of energy barriers. The quantitative measure of the relaxation is the magnetic viscosity defined as

$$
S=\frac{1}{M_{\mathrm{eq}}(H, T)} \frac{d M(H, T, t)}{d \ln (t)},
$$

where $M_{\mathrm{eq}}(H, T)$ is the equilbrium magnetization of the system at fixed temperature and field. The latter was deduced from the field cooled magnetization measurements.

As has been previously reported [2], at a small field $(H \sim 100 \mathrm{Oe})$ the viscosity is independent on temperature below $2.4 \mathrm{~K}$, pointing to the quantum origin of the low temperature magnetic relaxation in ferritin. This is in accordance with the temperature of the crossover from thermal to quantum regime, expected from the theory [3], $T_{c} \sim\left(2 \epsilon_{\mathrm{an}} \epsilon_{\mathrm{ex}}\right)^{1 / 2} / 2 \pi$. Here $\epsilon_{\mathrm{an}}=g \mu_{B} H_{\mathrm{an}}$ is the anisotropy energy, with $H_{\mathrm{an}} \sim 1 \mathrm{kOe}$ being the anisotropy field, and the exchange energy is defined as $\epsilon_{\mathrm{ex}}=J S$, where $J$ and $S$ are the exchange constant and the sublattice spin, respectively, in the antiferromagnetic interaction between sublattices, $J \mathbf{S}_{1} \cdot \mathbf{S}_{2} . H_{\text {an }}$ is defined as $2 K / M_{0}$, where $M_{0}$ is the magnetization of one sublattice [13,14]. The corresponding $\epsilon_{\mathrm{an}}=g \mu_{B} H_{\text {an }}$ represents the energy per spin that is needed to rotate the Néel vector away from the anisotropy axis, preserving the antiferromagnetic alignment of the sublattices. It is, therefore, the measure of the anisotropy barrier per spin. The actual magnetic field which is needed to rotate the moments of sublattices away from the anisotropy axis is much higher than $H_{\text {an }}$. It is the smallest of the two: $H_{\text {an }}^{\prime}=2 K / m_{0}$, where $m_{0} \ll M_{0}$ is the magnetization due to the noncompensation of sublattices, and $H_{f}=\sqrt{2 H_{\mathrm{an}} H_{\mathrm{ex}}}$, where $H_{\mathrm{ex}}=M_{0} / \chi_{\perp}$ and $\chi_{\perp} \sim 10^{-4}$ is the susceptibility with respect to the transversal field. The large field $H_{\text {an }}^{\prime}$ appears because the magnetic field couples to $\mathbf{m}_{0}$, not to $\mathbf{M}_{0}$. Another large field $H_{f}$, known as the spin-flop field, appears due to the coupling of $\mathbf{H}$ to the field-induced moment resulting from the rotation (resisted by the exchange) of the moments of sublattices towards the direc- tion of the field. The relation $H_{f} \gg H_{\text {an }}$ is known as the exchange enhancement of the anisotropy in antiferromagnets. We shall assume small noncompensation resulting in the antiferromagetic dynamics (see below). The value of the exchange field, $H_{\mathrm{ex}} \sim 10^{4} \mathrm{kOe}$ [7], can then be extracted from the measured value of the spin-flop field, $H_{f} \sim 100 \mathrm{kOe}$. In our notations, $\epsilon_{\mathrm{ex}}=g \mu_{B} H_{\mathrm{ex}}$. By order of magnitude $\epsilon_{\mathrm{an}} \sim 0.1 \mathrm{~K}$ and $\epsilon_{\mathrm{ex}} \sim 10^{3} \mathrm{~K}$, which provides the observed value of $T_{c}$. Note that the values of all parameters are in the right ballpark for that class of antiferromagnetic materials. Magnetic moments of ferritin molecules are produced by the noncompensated spin, $s=\left|S_{1}-S_{2}\right| \sim 50$. The antiferromagnetic dynamics takes place if the noncompensation is small, $s \ll S\left(\epsilon_{\mathrm{an}} / \epsilon_{\mathrm{ex}}\right)^{1 / 2}[1,15]$. This should be the case in ferritin where $s \sim 50$ while $S \sim 5 \times 10^{3}$. At $T<T_{c}$ the rate of quantum tunneling between different orientations of $\mathbf{s}$ is [3]

$$
\Gamma \sim \frac{T_{c}}{\hbar} \exp \left[-S\left(\frac{\epsilon_{\mathrm{an}}}{\epsilon_{\mathrm{ex}}}\right)^{1 / 2}\right] .
$$

With some inevitable uncertainties in the parameters, the temperature independent relaxation in ferritin in the limit of low temperature is consistent with the prediction of quantum theory given by Eq. (3).

The viscosity as function of the applied field at different temperatures is plotted in Fig. 3. At $T=2.4 \mathrm{~K}$ and $T=$ $3 \mathrm{~K}$ it increases monotonically with the field. Between 3 and $8 \mathrm{~K}$ the viscosity first drops as the field increases from zero, then reaches minimum at a certain field that depends on temperature, and then increases at greater fields. Above $8 \mathrm{~K}$ the viscosity monotonically decreases with the field. This is our main experimental finding that confirms the conclusion derived from the field dependence of $T_{B}$ and $d M / d H$ : in a certain temperature range the system relaxes faster in zero field than it does at a nonzero field.

This unusual behavior of the magnetic relaxation in ferritin can be explained if one takes into account quantization of spin levels in ferritin molecules. The low lying levels

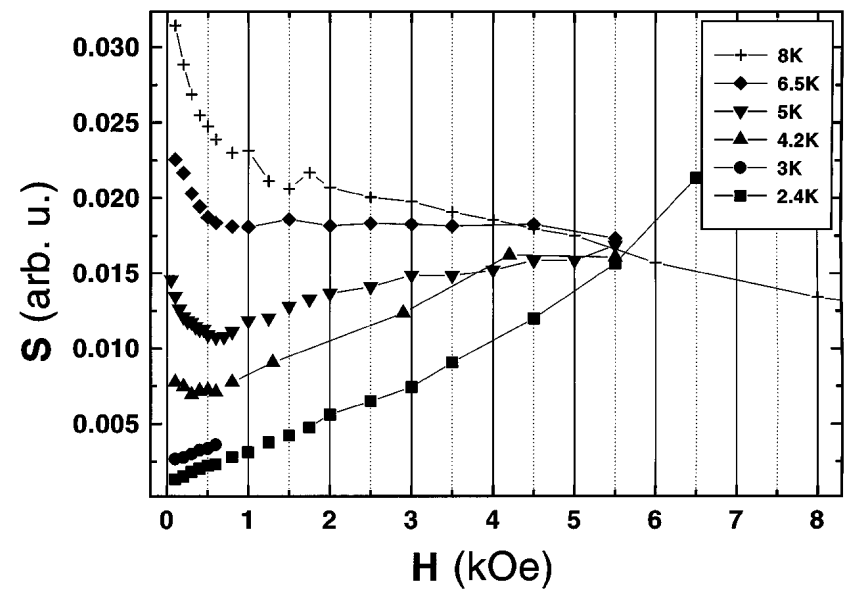

FIG. 3. Magnetic viscosity as a function of the magnetic field at different temperatures. 
must be separated by the energy of the antiferromagnetic resonance, $\epsilon=\left(2 \epsilon_{\mathrm{an}} \epsilon_{\mathrm{ex}}\right)^{1 / 2}$, which is huge compared to the separation of spin levels in a ferromagnetic particle of a comparable size. In ferritin this separation must be of order of $10 \mathrm{~K}$, that is, comparable to the separation of low lying levels in Mn-12 molecules of spin 10. This suggests that the resonant spin tunneling recently observed in $\mathrm{Mn}$ 12 molecules [16] can also be observed at a macroscopic level in ferritin molecules containing a few thousand iron atoms. The argument goes as follows: Consider an individual ferritin molecule in the magnetic field $\mathbf{H}$ opposite to its total spin $\mathbf{s}$. This is a metastable state that wants to decay towards the direction of the field. The resonant tunneling between the spin level occurs when $2 g \mu_{B} H s=n \epsilon$, where $n=0,1,2, \ldots$, that is, at values of the magnetic field separated by

$$
\Delta H=\frac{H_{\mathrm{an}}}{2 s}\left(\frac{2 \epsilon_{\mathrm{ex}}}{\epsilon_{\mathrm{an}}}\right)^{1 / 2} .
$$

For a ferromagnetic particle of spin $S$, the field separation between resonances would be $H_{\text {an }} / 2 S$ [10], which for $H_{\text {an }} \sim 1 \mathrm{kOe}$ and $S \sim 10^{4}$ gives $\Delta H \sim 0.1 \mathrm{kOe}$. This would certainly be smaller than the width of the levels (the ferromagnetic resonance width) and, thus, unobservable. For an antiferromagnetic particle, however, due to the smallness of the noncompensated spin and the exchange enhancement of the anisotropy, the field spacing of resonances is greater by a factor $(S / s)\left(\epsilon_{\mathrm{ex}} / \epsilon_{\mathrm{an}}\right)^{1 / 2}$, which is of the order of $10^{4}$ in ferritin. Consequently, the field spacing between resonances in ferritin must be of order of $\mathrm{kOe}$, that is, comparable to that in Mn-12. However, due to the different orientation and magnitude of the noncompensated spin, contrary to the case of a Mn-12 crystal, only the $H=0$ resonance occurs simultaneously in all ferritin molecules.

As in Mn-12, tunneling between different spin orientations in ferritin in our temperature range must be thermally assisted; that is, it occurs from excited spin states as well as from the ground state. The spin states are characterized by the projection of $\mathbf{s}$ on the anisotropy axis, $s_{z}|m\rangle=m|m\rangle$. At $H=0$ the states corresponding to $m$ and $-m$ have exactly the same energy and the resonant tunneling between these states takes place. At low temperature (below $3 \mathrm{~K}$ ) only the lowest levels $m= \pm s$ are occupied. The tunneling splitting of these levels is $\Delta=\hbar \Gamma$, where $\Gamma$ is given by Eq. (3). It determines the width of the resonance on the magnetic field. Since tunneling is weak this width is extremely small. According to Ref. [1] where measurements were performed in the $\mathrm{mK}$ range, the width of this resonance is about $10^{-4}$ Oe. This should explain why we do not see the $H=0$ peak in the viscosity at low temperature. As the temperature increases above $3 \mathrm{~K}$, higher $m$ levels become thermally populated and tunneling between them dominates relaxation. The width of these levels must be large due to their finite lifetime with respect to the decay down to the ground state levels $m= \pm s$. Consequently, the width of the resonance on the magnetic field becomes large enough to observe the $H=0$ maximum in the magnetic viscosity. As the temperature continues to increase towards the blocking temperature, the system enters the superparamagnetic regime dominated by thermal overbarrier transitions. In this regime the viscosity monotonically decreases with the field, reflecting the progressive disappearence of the metastability

In conclusion, we have presented data on the magnetic relaxation in ferritin which show strong departure from the conventional superparamagnetic behavior. A plausible explanation to all data is given within the model of thermally assisted resonant tunneling between spin levels. Our data seem to support the suggestion first made in Ref. [1] that ferritin exhibits quantum tunneling on a macroscopic scale.

J.T., X.X.Z., and J.M.H. acknowledge support from CICYT Project No. IN96-0027 and CIRIT Project No. 1996-PIRB-00050. The work of E. M.C. has been supported by the U.S. National Science Foundation Grant No. DMR-9024250. E. D. B. acknowledges support from the University of Barcelona.

[1] D. D. Awshalom et al., Phys. Rev. Lett. 68, 3092 (1992).

[2] J. Tejada and X.X. Zhang, J. Phys. Condens. Matter 6, 263 (1994).

[3] B. Barbara and E. M. Chudnovsky, Phys. Lett. A 145, 205 (1990).

[4] J.L. van Hemmen and A. Sütö, Physica (Amsterdam) 141B, 37 (1986); Europhys. Lett. 1, 481 (1986).

[5] M. Enz and R. Schilling, J. Phys. C 19, 1765 (1986); 19, L711 (1986).

[6] E. M. Chudnovsky and L. Gunther, Phys. Rev. Lett. 60, 661 (1988).

[7] S. Gider et al., Science 268, 77 (1995).

[8] J. Tejada, Science 272, 424 (1996); Anupam Garg, Science 272, 424 (1996); S. Gider, D. D. Awschalom, D. P. Divincenzo, and D. Loss, Science 272, 425 (1996).

[9] S. Gider et al., J. Appl. Phys. 79, 5324 (1996).

[10] J. R. Friedman et al. (unpublished).

[11] R. Sappey et al. (unpublished).

[12] S. H. Kilcoyne and R. Cywinski, J. Magn. Magn. Mater. 140-144, 1466 (1995).

[13] C. Kittel, Quantum Theory of Solids (Wiley, New York, London, 1963).

[14] A.H. Morrish, The Physical Principles of Magnetism (Krieger Publishing Co., Malabar, FL, 1983).

[15] E. M. Chudnovsky, J. Magn. Magn. Mater. 140-144, 1821 (1995).

[16] J. R. Friedman, M.P. Sarachik, J. Tejada, and R. Ziolo, Phys. Rev. Lett. 76, 3830 (1996). 